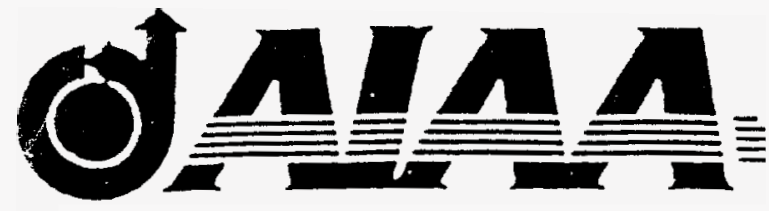

\section{AIAA 92-0409 Numerical and Experimental Analysis of Vortex Sheets Behind Lifting Surfaces}

M. P. Warzecha

Boeing Commercial Airplane Group Seattle, Washington

H. H. Horng

University of Michigan Ann Arbor, Michigan

\section{0th Aerospace Sciences Meeting \& Exhibit January 6-9/1992/ Reno, NV}




\title{
Numerical and Experimental Analysis \\ of \\ Vortex Sheets Behind Lifting Surfaces
}

\author{
M. P. Warzecha* \\ Boeing Commerial Airplane Group \\ Searde, Washington \\ H. H. Homg** \\ University of Michigan \\ Ann Arbor, Michigan
}

\begin{abstract} tial applications exist for this type of model.

\section{Nomenclature}

L' sectionallift

$U_{\infty}$ teestream velocity

b wingspan

c chord leagth

$i, j$ indices for point vortices

$s$ direction along yortex sheet

$u_{i}$ induced $z$-yelocity in Tretrz plane

$v_{i}$ inducedy-yelocity in Trefftz plare

$x$ downstream direction

$x / b$ nomalized downstream direction

y direction normal to $z$ in the Treffiz plane

$z$ seanivise direction

$\Gamma$ ' local bound circulation

$\Gamma(s)$ filament strength distribution

$\delta$ vortex sheet thickness

$Y(s)$ circulation density distribation

$P$ density

(1) yorticity
\end{abstract}

Vortex wake phenomena are crucial to moders aircraft design and operation. Based on an established theory, this study presents a computational model of the vortex sheet evolution behind lifting surfaces. Furhermore, experiments are conducted to as sess the numerical results of this study. The computational model is applied to understand how alterationsin olanform geametry affect engine exhaust dispersion rate. Many other poten-

- Bayload Destign Engicer Boeing Commercial Airplane Group Member AIAA

* Graduate Suden: The Uniyersity of Michigan Student Member AIAA

Copytight (1) 1992 by the Anerican Instute of Aeronautics and Astronaudics, Inc. Ail rights reserved.

\section{Introduction}

Until reenty, trailing yorex sheet research depended heavily on costly wind nunnel and flight tests. With the advent of digital computers, panel methods were used to model the 3-D vortex wakes behind aircraft. However, such 3-D panel methods require extensive computational effort This investigation utilizes a 2-D vortex method to develop an accurate and inexpensive computational model of the trailing vortex sheet. This method was previously studied by Rosenhead (5] and Chorin and Bemard [1].

The und rstanding of airplane vortex wake phenomena is critical to commercial and military aviation. Powerful wake vortices generated by passenger ai-liners and transport aircraft are extreacely bazardous, especially during take-off and landing. Air traffic must be spaced to avoid accidents. The direct consequence is that landing and takeffir rates are re s a i d below maximum aipport capacity.

There are benefits from utilizing vortex wake phenomena. Military applicationsof $v$ a t a wakes include increasing airplane stealthiness through the trake mixing of hot engine exhaust. Studying vorter fiates may also help to develop methods of reducing induced drag.

In this investigation, the computational model represents the wake behind a lifting surface by a series of span rise vortex filaments. Circulation streagth distribation is dictated by the planform. A fourth-order Runge. Kuta integration schewe computes the wate evolution in incremental Trefftz-plane behind the lifting body. To avoid aumerical instability, a smoothing parameter is used to desingularize the governing equations [4]. The YB-49 and the B-2 wen chosen for this study because their close approximation to a lifting siriace. Finally, experiments were performed to asses numerical results.

\section{Theory}

According to classical ratyomics, an airfoil section can bodeded as a bound varter th a sectional lift L'(y) given:

$$
L^{\prime}(y)=p U \Gamma(y)
$$

Assuming the olanform to be untwisted and composed of selfsimilar airfoils, the sectional lift is directly proportional to the airfoil chord longt:

$$
L^{\prime}(y) \propto c(y)
$$


Since the sectional circulation is directly propomonal to lift, the local bound circulation strength is also propomonal to the chord length:

$$
\Gamma(y) \propto c(y)
$$

The change in bound circulation between adjacent sectiona airfoils must form a vortex filament in the streanwise direction. The filament circulation density is qual to the difference in circulation between the adjacent differential wing

$$
\gamma(y) \propto d \Gamma d y
$$

Across the entire span of the lifting surface, this forms a vortex sheet composed of infinite filaments; therefore, the circulation density of each filament is propomonal to the local change in chord length. The individual filament circulation strength is given by:

$$
\Gamma(y)=\gamma(y) d s
$$

where $d s$ is the width of an individual vortex filament. By substitution, the circulation stsength of each filament is given by:

$$
\Gamma(y)=\left(\frac{d c}{d y}\right) d s
$$

For this investigation, the governing equation is the conservation of momentum expressed in tems of the vorticity field, Equation (7):

$$
\left[\frac{\partial}{\partial t}+u \cdot v-\frac{1}{R e} \nabla^{2}\right] \omega(x, t)=\omega \cdot v u
$$

Since the compressibility effects are negligible in the railing wake, this form of the momentum conservation essentially determines the evolution of the vortex sheet. Furthermore, two additional assumptionsapply in this case. For typical aircraft applications, the diffusivity $\left(1 / \boldsymbol{R}_{\boldsymbol{e}}\right)$ is small: therefore, the diffusion term can be neglected. As a consequence, there will be no physical elimination of vorticity due to mixing. The steady three-dimensional trake can be approximated with an unsteady rwo-dimensional flow in the Treffz plane. This car be justified by the fact that the wake evolves faster in the Treffez plane as compared to the downstrean direction. Hence, the stretching tern, $\boldsymbol{\omega} \cdot \nabla_{\mathbf{l}}$ disappears. Therefore, the following induced ve. locities are derived:

$$
\begin{aligned}
& \frac{d x_{1}}{d t}=\sum_{i \rightarrow j} \frac{\Gamma_{i}}{2 \pi} \frac{-\left(y_{i}-y_{i}\right)}{\left(x_{1}-x_{i}\right)^{2}+\left(y_{1}-y_{i}\right)^{2}+\delta^{2}} \\
& \frac{d y_{1}}{d t}=\sum_{i \rightarrow j} \frac{\Gamma_{1}}{2 \pi} \frac{\left(x_{1}-x_{i}\right)}{\left(x_{i}-x_{i}\right)^{2}+\left(y_{i}-y_{j}\right)^{2}+\delta^{2}}
\end{aligned}
$$

To avoid numerical instability and singularity, the smoothing parameter, $\delta$, is inserted ( $1 \& 4$ ]. Physically, $\delta$ represents the finite thickness of the vortex sheet

\section{Numerical Model of the Vortex Sheet}

As mentioned previously, a yortex sheet can be analytically modeled as an infinite series of vortex filaments, each with a cir culation density determined by the local rate of change in chord length. Computationally, the vortex sheet is modeled as a finite series of filaments, each with a width of ds. With the stated assumptions, the computer performs the unsteady two- dimensional calculations in Trefftz planes behind the lifting body. All displacements are nommalized by the wingspan. At each Trefftz plane, the induced velocities of all vortex filaments are calculated and summed using Equation (8). Using the forthorder Range-Kura integration scheree, each point is advanced to the next Trefftz plane. This marching procedure represents the sheet evolution in tree.

As the wake calculation progresses, the spacing between individual vortex filaments increases; therefore, additional points are insered to insure adequate filament resolution. If the displacement between adjacent filaments exceeds a predefined value, Newton's polynomial interpolation is utilized to approximate the shape of the vortex sheet. The circulation strengths of the inserted point and its reighboring points are adjusted accordingly. Conversely, high concentration of filaments occur in spe. cific areas of the vortex sheet. Extra computation docs not justify the limited sheet description gained fom excessively high fil. ament concentration Funure program modifications may include a point-ranovalschetxe. Before implementing a full scale analysis, three test cases involving point-vortex pairs were conducted to insure program integrity,

\section{Numerical Results}

For this investigation, two aircraft are chosen for fill scale analysis of vortex sheet evolution, the Northrop YB-49 and the Norhrop B-2 Steal th Bomber. The aircraft are flying wings that can be represented as lifting surfaces fining the scope of this study.

\subsection{YB-49 Planform}

The YB49 provides a simple delta-wing planform[2] with a knownvortex stortution. hence it is useful in verifying the full scale implementation of the computational model. The vortex sheet of the YB-49 can also be used as a comparison for the B-2. The verical stabilizers and the suall fuselage section arc igaored due to their minimal contribution to the vortex sheet. The planiorm of the $Y B-49$ and the corresponding circulation density distribution are shown in Figure 1. In the this plarform. it is worthy to note the infinite circulation density values at the wing tip caused by the chord-length discontinutites.

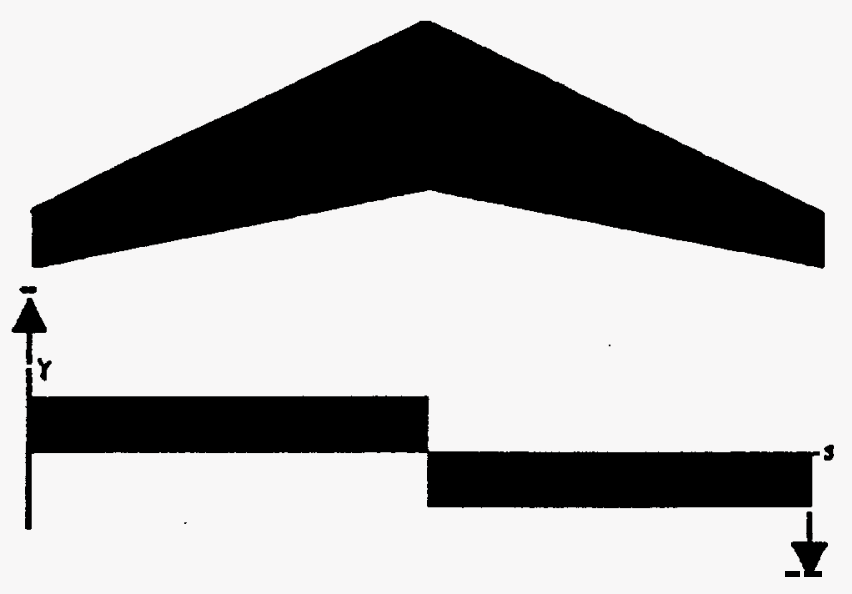

Figure 1 YB-49 planform and circulation density distribution

As expected, the simple delta-wing planform of the YB.49 produces a pair of counter-rotating yorices. Figre 2 shows the vortex sheet evolution of the YB49 at various normalized dowrstream locations. 

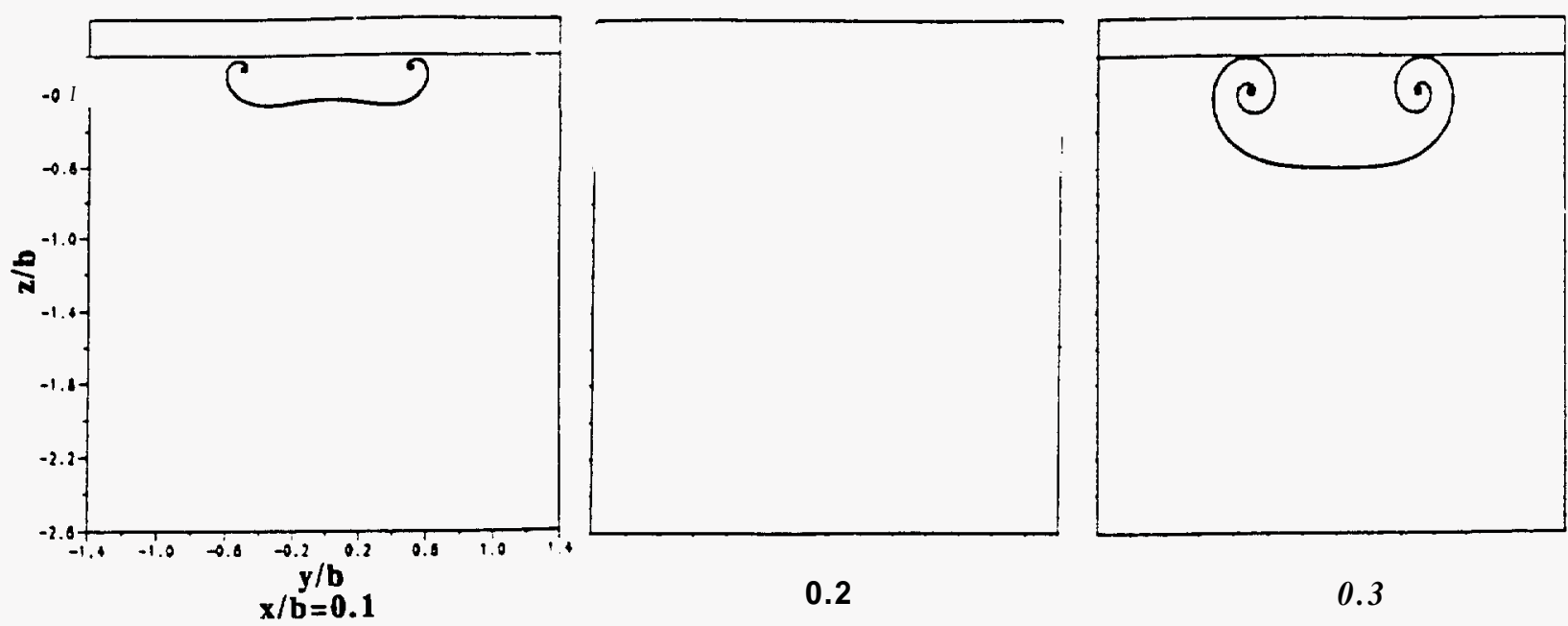

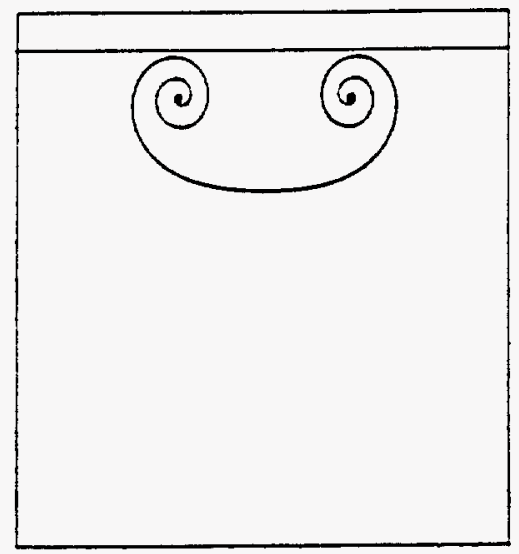

0.4

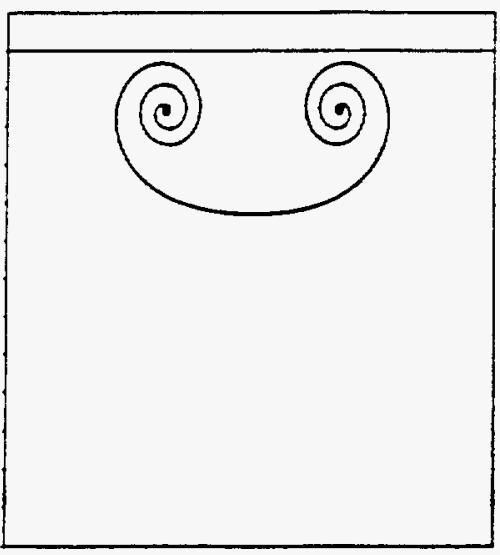

0.5
0.3

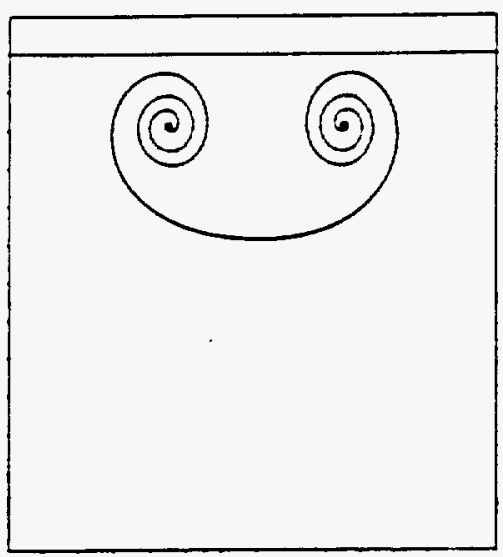

0.6

Figure 2 YB.49 vortex sheet evolution

\subsection{B-2 Planform}

The B-2 has a more incerestiog planform[6]. Although there are no chord-length discontinuities in this planform, the "sawtooth-like" trailing edge provides an unique, yet sorerthat regu. lar, circulation density distribution shown in Figure 3 .

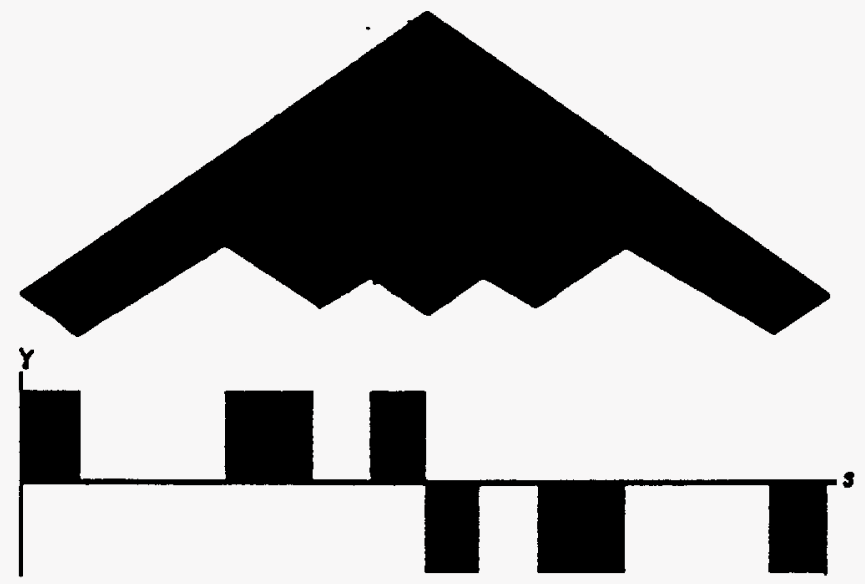

Figure 3 B-2 planform and circulation density distribution
The yortex sheet evolution of the B-2 does not resemble a conventional roll-up. The unique circulation density tistribution

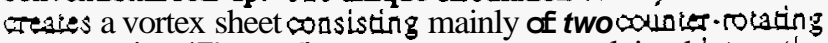
vortex pairs (Figure 4). For a purpose explained later, the points corresponding to be engine exhaust are matxed with cir. cles. Note that at a significas downstreamdistance the yortex sher volves into a counter-rotating yorex pair.

\subsection{B-2 Modifications}

This vortex tracking technique is applicable in many areas of aircraft design and fluid dynamics. For example, this technique may be used to produce a first-order approximation of exhaust detectability and to provide insight into planform modifications to reduce the detectability. As part of the investigation, this example concentrates on how the vortex sheet evolution disperses the engine exhaust and improves dispersion rate. One must keep in mind that ocher design features also contribute to the dispersion of exhaust gas.

The de followity approximation includes the sumptions. The detoctability of a passive scalar in the trailing wake,e.g. the thermal energy introduced by the exhaust, is inyesely progortional to the rate at which the engine arc-length increases. "Engine arc-length" is defined as the length of the arc forred by the exhaust points along the s-direction $a$ any normalized do grastream locations. Throughout the vortex sheet evolution, the points corresponding to engine exhaust arc individually marked by a circle. Furthermore, to avoid difficulies in 


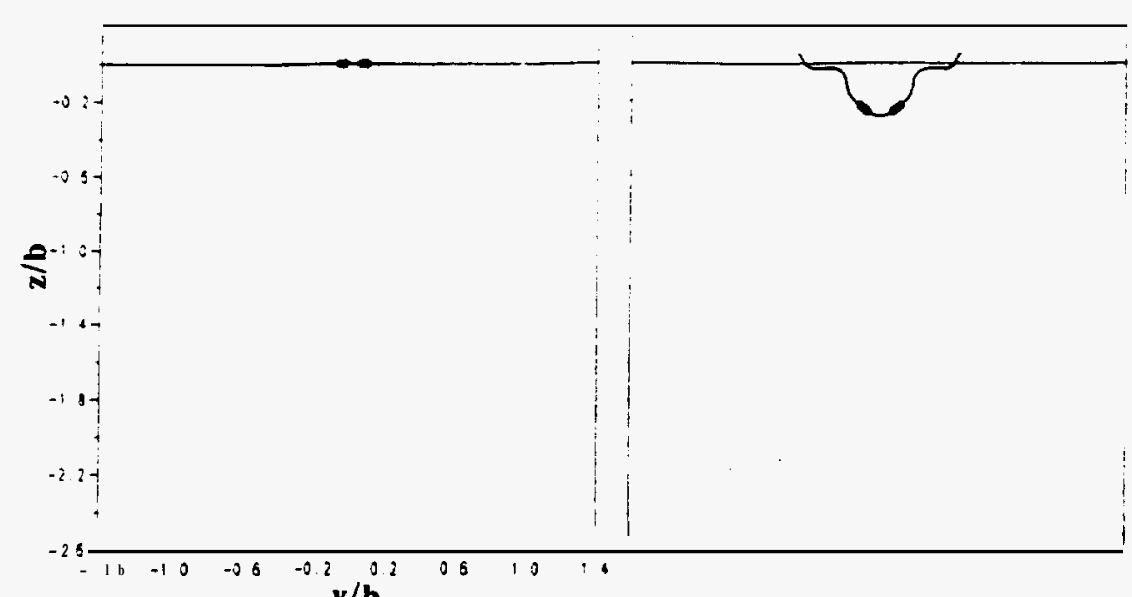

$\boldsymbol{y} / \mathbf{b}$

$x / b=0.0$

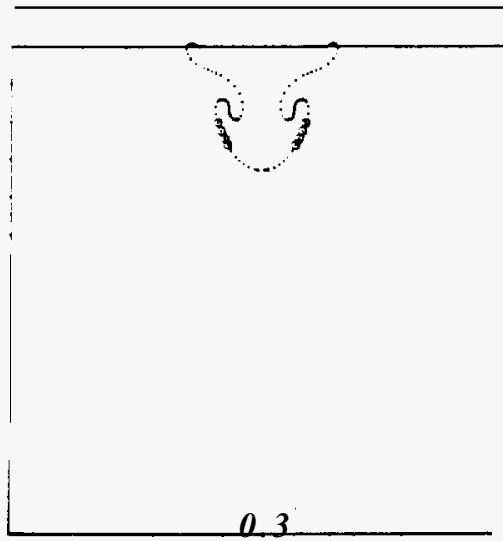

0.3

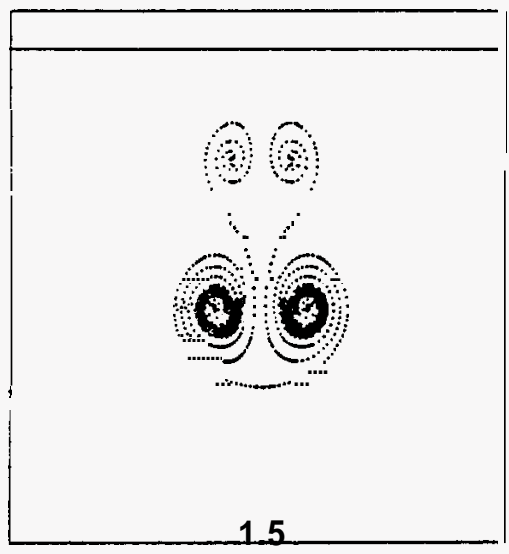

1.5
0.1

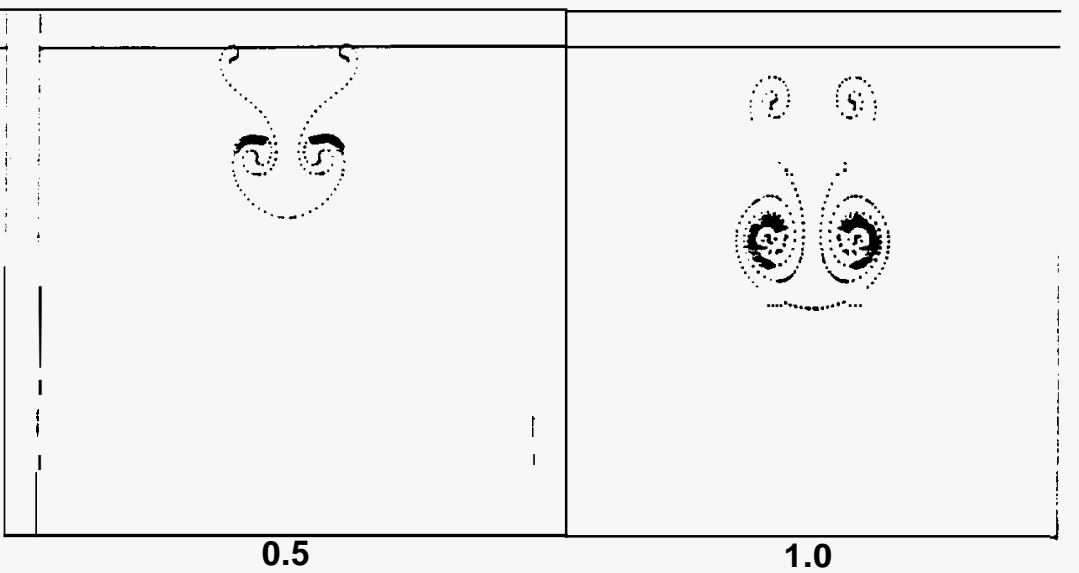

0.5
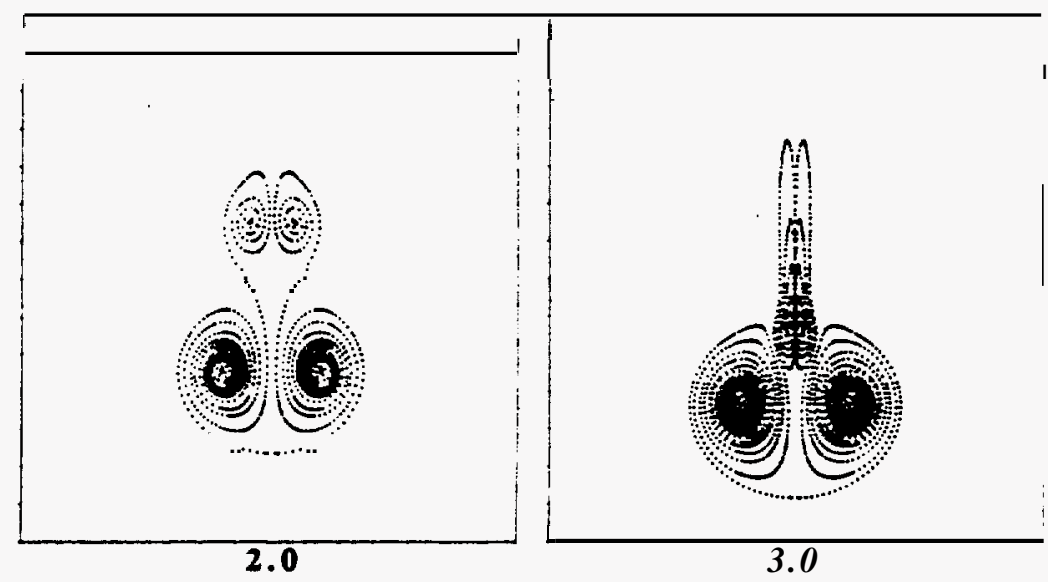

Figure 4 B-2 vortex sheet evolution

wodeling the complex mixing process between exhaust and ambient air, it is assumed that the exhaust velocity equals the freestream velocity.

Several planform deviations from the original B-2 are studied in an attempt to understand how changes in the circulation density distribution, $\gamma(s)$, affects the dispersion rate. To reduce the impact on other design considerations, minimal changes are made in each planform modification.
Tut most important insight obtained is that drastic changes in $\gamma(s)$ the exhaust nozzles are most effective in increasing $p$ dispersion rate. The modified planform with the greatest increase in dispersion rate, designated as the YB-2EF, is shown in Figure 5 with its circulacion density distribution. 

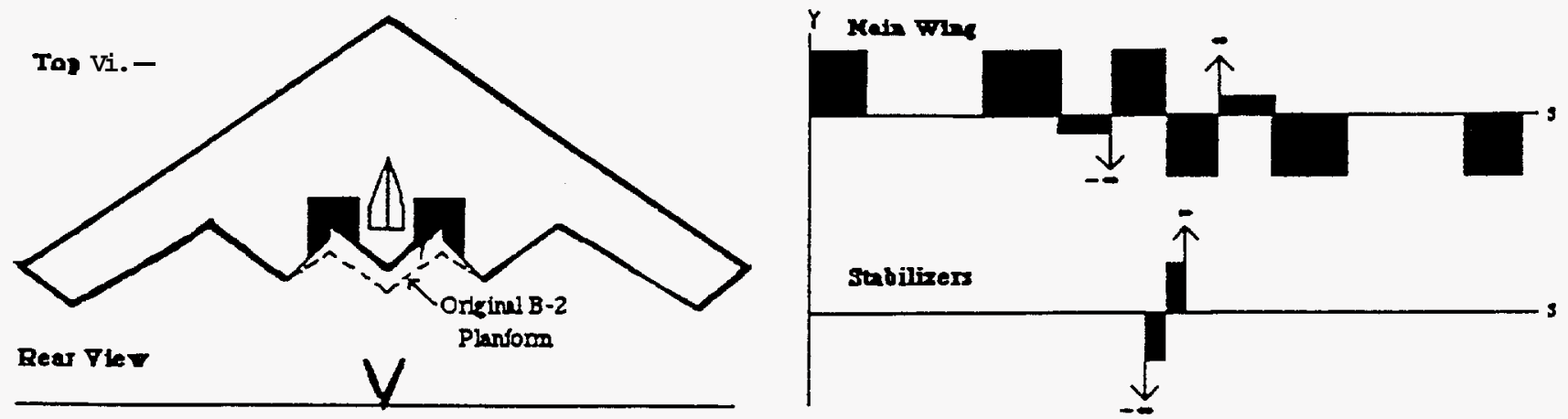

Figure 5 YB-2EF planform and circulation density distribution
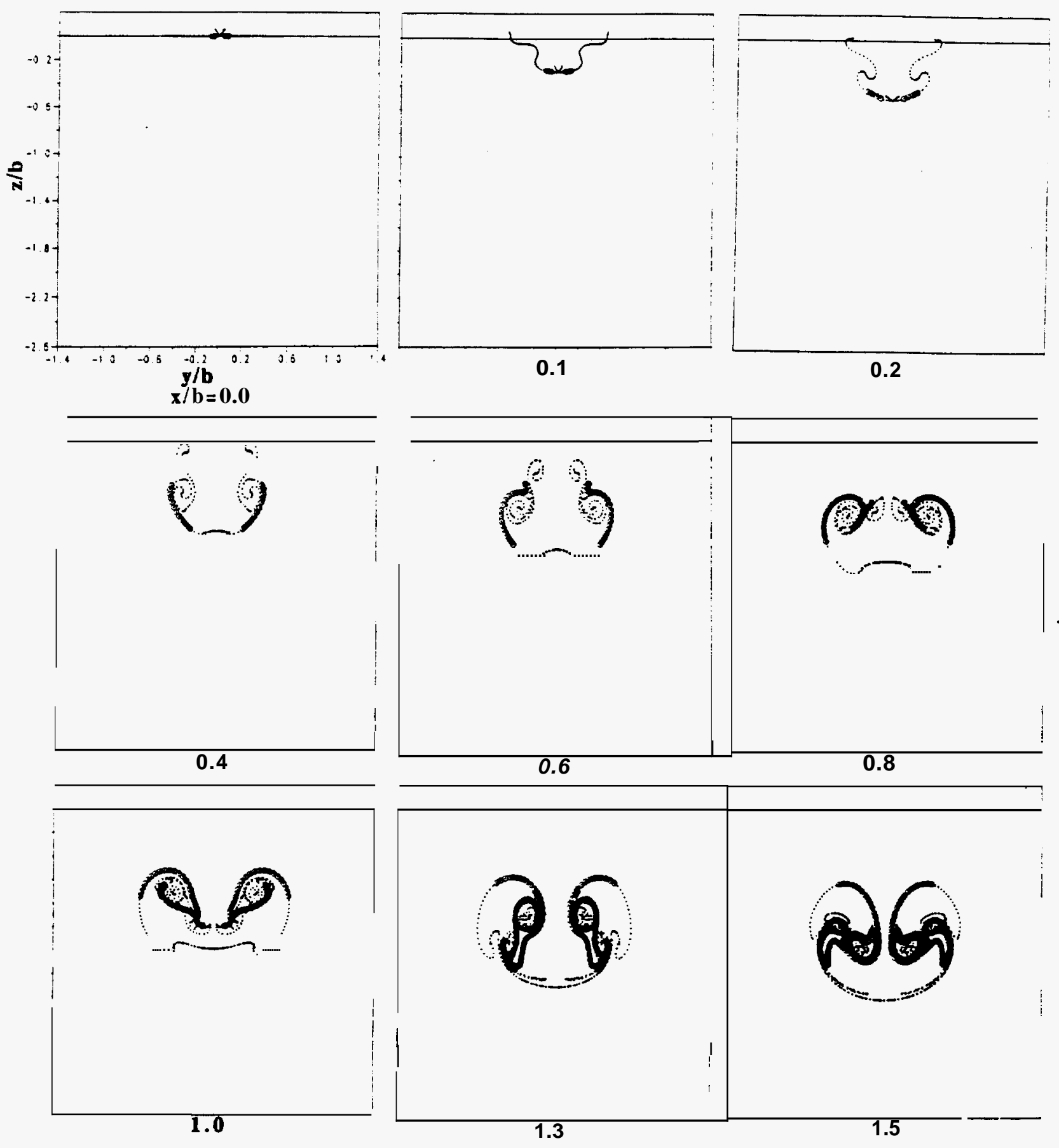

Figure 6 YB-2EF vortex sheet evolution 
The Vtail is placed along the centerline with a $70^{\circ}$ included angle similar to the F-117. The included angle prevents the tail from acting as a come radar reflector[3]. Unlike the rest of the planform, the Vtail produces negative liff which results in a negative $\gamma(\mathrm{s})$ for the left tail and a positive value for the right. The tips of the V-tail provide theoretically infinite circulation density values due to the discontinuities in the chord length rate of change.

As compared to the B-2. the circulation density for the center-most section of the $Y \bar{B} \cdot 2 E F$ is unchanged. By changing the trailing edge slope of the next outboard sections, dc/dy becomes negative for the left outboard section and positive for the right outboard section. Although the circuation density value remains the sarve for the center-most section, the actual chord lengths are shortened to introduce mother set of theoretically infinite circula tion density values at the centers of the exhaust nozzles. This modification plays an important role in increasing the dispersion rate. Figure 6 demonstrates the vortex sheet evolution of the YB.2EF, and Figure 7 shows a direct comparison of the engine arc-length growth rate between the YB-2EF and the B-2. Although the modifications may have reduced the thermal de. tectability, vulnerability of aircraft detection by other means and manufacturing difficulties may have been simultaneously increased.

\section{Experimental Assessment}

The experimental portion of this investigation was conducted at the University of Michigan Aerospace Engineering Deparroent towing tank facility. The experimental results assess the accuracy and validity of the computational nodel.

The experiment consists of towing a scaled B-2 model in a water tank The model is mounted a approximately $12^{\circ}$ angle of attack and supported at the centerline. As the model travels downstream, gravity-fed laser fluorescent dye emerges from a milled opening on the pressure side of the model. A neon-argon laser beam, oscillating at approximately $180 \mathrm{~Hz}$, produces a laser sheet normal to the freestream, analogous to the numerica\} Trefftz-plane. The laser sheet excites the dye molecules and reveals the two-dimensional structure of the vortex sheet, enabling a camera to photograph the evolution

Figure 8 shows the various frames of the experimental vortex sheet evolution. Two separate mals are shown, Frames (a) through (d) and Frames (e) through (h). In (a) through (d), the initial vortex sheet development is clearly shown. In this trial nun. the dye passage was partially blocked causing the missing section in the right half of the vortex sheet

Although the B-2 is the only planform tested in the towing tank, the results were sufficient to assess the validity of the numerical model. The general shape of the vortex sheet at various downstream locations is in close agreement with the numerical output As demonstrated by the experiment, the upper vortex pair collapses and mixes wore rapidly with the lowervortex pair. This mixing phenomenon would have obtained numerically if the computations were performed to a farther $x / 0$. In addition, Frames (e) and (f) show that the vortex sheet evolves into a pair of counter-rotatingvortices, as stated arier

$$
x=Y B \cdot 2 E F \quad 0=B-2
$$

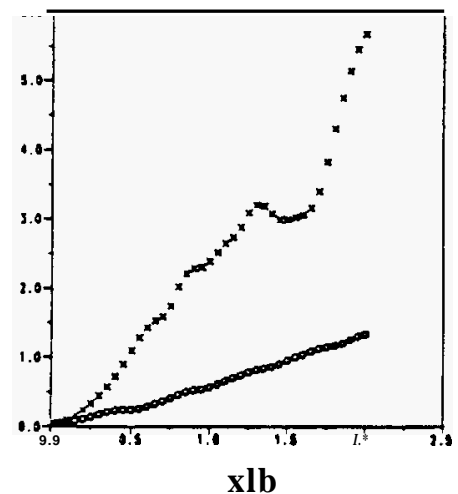

Figure 7 (a) Comparison of engine arc-length growth rate between $\mathrm{YB}-2 \mathrm{EF}$ and $\mathrm{B}-2$

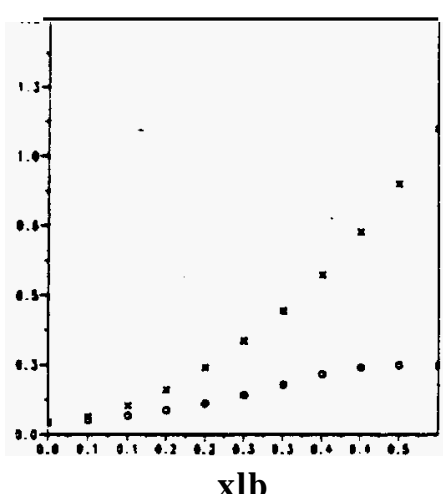

Figure 7 (b) Comparison of the early stages 


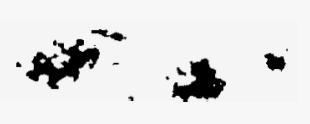

(a)

1
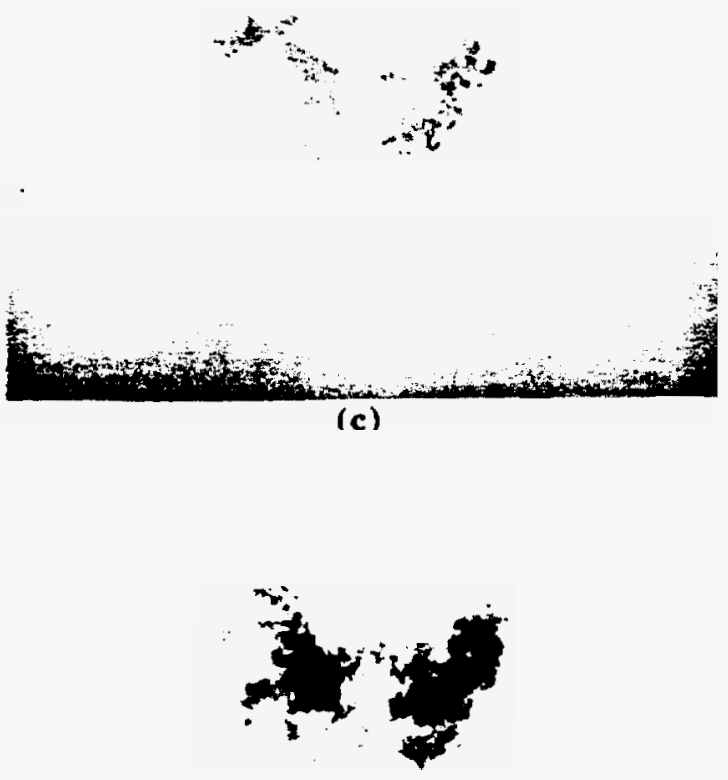

(e)

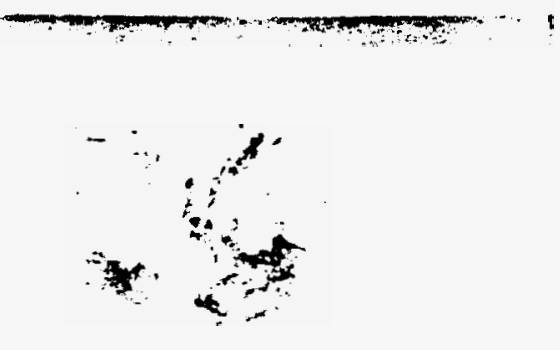

(g)
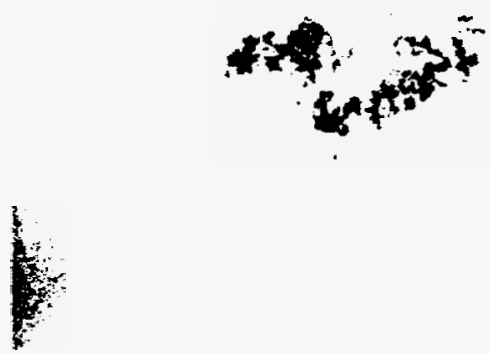

(b)
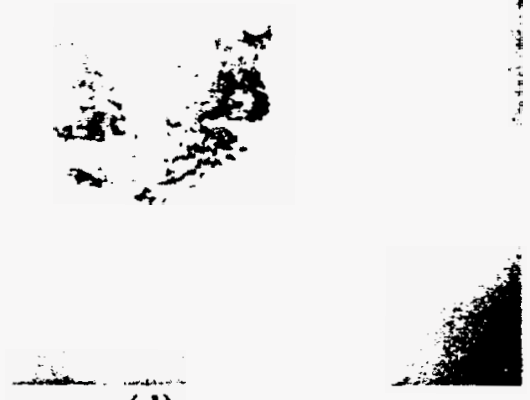

(d)
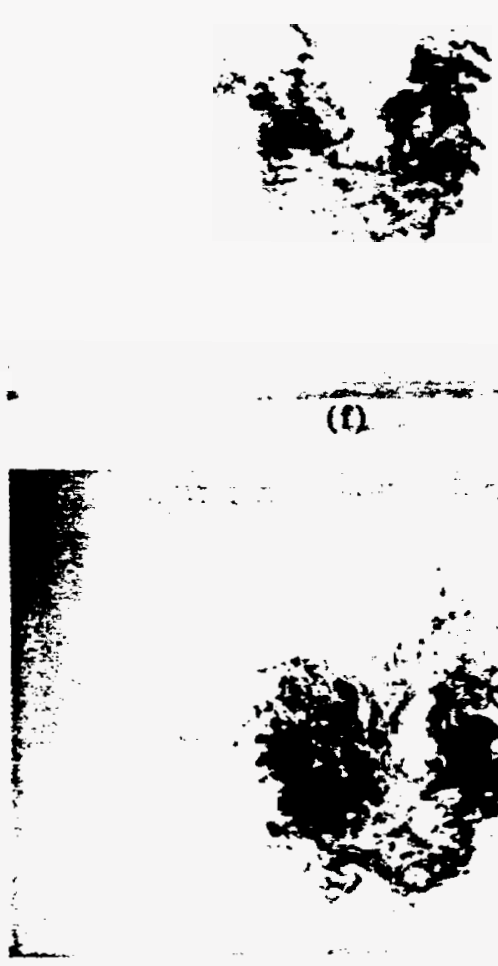
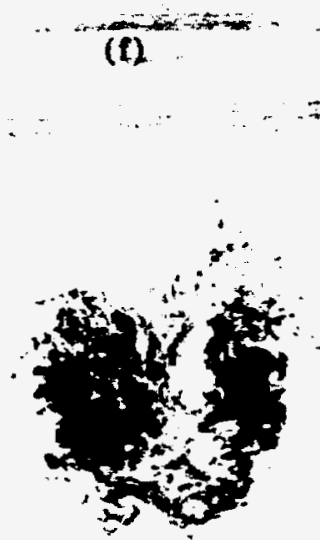

(h)

Figure 8 B-2 experimental vortex sheet evolution 


\section{Conclusions}

This study has developed a simple numerical model of lifting body vortex wakes. Two aircraft are used for full scale implementation, the YB-49 and the B-2. The aircraft are chosen for their close resemblance to lifting bodies. Experimental data demonstrated that the computational model satisfactorily simu lates the vortex sheet evolution.

This investigationutilizes the computational model to obtain an approximation of the exhaust dispersion phenomenon. Modifications are made to the B-2 planform to understand how the exhaust dispersion rate is affected by planform geometry. One major observation is made from the modifications. Discontinuities placed near the exhaust nozzles are significant factors in increasing the dispersion rate. An important conclusion from this example is that small modificacions in planform geometry can considerably alter the vortex wake evolution and the corresponding dispersion rate.

\section{Future Applications}

Before applying the vortex tracking technique to more complicated problems, other topics must be addressed in order to make this a more complete tool. The topics include: presence and effects of the fuselage and empennage on the vortex sheet, ground effect, and three-dimensionalmixing of the exhaust air.

To more accurately model an airplane, the presence of the empennage and the fuselage must also be considered. The empennage control surfaces can be modeled with the same yortex racking technique; however, fuselage effects require a more complex technique. To simulate take-off and landing conditions, the ground effect can be modeled with the method of imaging. In addition, cross-wind effect can be simulated by assigning a pre-specified induced velocity on the flaments. In reality, the aircraft exhaust velocity does not equal the irestrean velocity. Therefore, the three-dimensional mixing effects of the exhaust must be considered to wore accurately model dispersion phenomenon.

In addition to the example application investigated, this vortex racking technique is also useful in other areas of fluid dynamics and aircraft design. Furter understanding of the trailing wake phenomenon can assist in optimizing the aimor take-off and landing rates. Other military applications exist for this tect. nique. In addition to reducing the aircraft thermal detectability. this technique may also be applied to reduce the trailing wake disturbance introduced. A major concern in submarine pariare is the detection of free surface signaure produced by the control surfaces. This technique may aid in reducing the free surface signature.

\section{References}

1. Chorin, A.J. and Bernard, P.S. "Discretization of a Yortex Sheet, with an Example of Roll-Up,"J. Computational Physics, 1973, Wol.13, pp. 423-429.

2. Coleman, T., Jack Northrop and the Flying Wing. New York Paragon House, 1988.

3. Domheim, MA., "USAFDisplay of F-l17A Reveals New Derails of Steal th Aircraft,"Aviarion Week and Space Technology April 30,1990: 31

4. Krasny, R, "Desingularizadon of Periodic Wortex Sheet Roll-Up,"J. Computational Physics, 1986, Vol.65, pp.292-313.

5. Rosenhead, L, "The Formation of Vorticies From a Surface of Discontinuity," Proc. Royal Sociery of London, 1931, bl.134. p.170.

6. Sweernan, B. Stealth Bomber. pp. 91-102. Osceola: Motorbooks Int, 1989.

\section{Acknowledgments}

This project was conducted under the guidance of Professor W.J.A. Dahm, the University of Michigan Deparment of Aerospace Engineering. 